\title{
Editorial
}

\section{Topical collection on Chromatin Biology and Epigenetics}

\author{
Published online: 8 January 2020
}

At an Indo-US conference held in Bengaluru in 2018, we were discussing the many opportunities for collaborations between scientists in the US and India. We realized that having a regular meeting in India akin to a Gordon conference would provide the continuity to foster such collaborations and also enable experimental workshops and courses analogous to those at Woodshole and CSHL, USA. As a first step, a hands-on laboratory based course on 'Phase Separation in Genome Organization' was conducted by Geeta during July-August 2019 at IISER Pune. This course was attended by 15 participants that included $\mathrm{PhD}$ students and postdoctoral fellows from various institutions in India.

As another step we felt it would be useful to put together a special issue of the Journal of Biosciences centered around the theme of Chromatin Biology and Epigenetic Regulation. The Editors of the journal enthusiastically supported this initiative. Our aim was to assemble a collection that consisted of topical reviews by leaders in the field as well as reports of original research or analysis. This issue represents the results of these efforts and includes contributions primarily from scientists in US and India. Below we provide a brief preview of the collection.

Among the diverse modes of transcriptional regulation, regulation dictated by chromatin organisation is immensely critical, albeit insufficiently understood. The reorganisation of chromatin in three dimensional space inside a eukaryotic nucleus can allow discrete regulatory regions of the genome to make or break contacts leading to formation of transient regulatory hubs wherein supramolecular complexes can assemble and regulate gene expression patterns. A number of exciting findings centered around these themes have been accumulating in recent years and work related to many of these themes is featured in this special issue. These include histone isoforms and post-translational modification marks; transcriptional regulatory tools- histone readers, writers and erasers; DNA methylation and the different tiers of chromatin organisation. The growing relevance of these mechanisms is signified by the diversity of models systems used in these studies, which range from the simpler forms, including bacteria, yeast, Plasmodium falciparum (the malarial parasite in humans), to systems such as Hydra vulgaris (fresh-water polyp), Drosophila (and other insect systems) and human.

Research article by Mukherjee and Sen et al. (Chamdrima Das's group) highlights the 'dual-reader' activity of the tumor suppressor protein ZMYND8 by binding to H4K16Ac and H3.1K36me2 marks and regulating differentiation genes, further bolstering the scope of differentiation therapy in cancer treatment. The article by Pant et al. (Sanjeev Shukla's group) reports a 34 gene 'hypoxia signature' to identify hypoxic and normoxic tumors, on the basis of which, they have been able to identify two differential spliced genes (DHX32 and BICD2), specifically due to altered intragenic DNA methylation in tumors. Genomic imprinting through parent-of-origin dependent DNA methylation marks is known to occur in a few insect species, plants, and placental mammals. Studies on imprinted genes have contributed immensely to highlighting the role of reversible epigenetic modifications and the epigenetic circuitry during gene expression and development. In their review article on the role of genomic imprinting in mammalian development, Thamban et al. (Sanjeev Khosla's Group) discuss the phenomenon of genomic imprinting in mammals and the roles played by it especially during fetoplacental growth and

This article is part of the Topical Collection: Chromatin Biology and Epigenetics. 
early development. Lio et al. from Anjana Rao's group review the findings in the field of TET (Ten-Eleven Translocation) family hydroxymethyltransferase biology within the last decade, since the discovery of their biochemical activity. They elaborate on how TET proteins, through $5 \mathrm{hmC}$ modification, regulate the function of enhancers and loss of TET function is associated with cancer.

Birch and Shilatifard (Ali Shilatifard's group) have reviewed the 'dynamic chromatin states' dictated by histone modifications in the context of leukemia, which can aid in diagnostic and therapeutic strategies. In their review article on the regulation of epigenetic states by non-histone chromatin proteins and transcription factors, Sikder et al. (Taps Kundu's group) have focussed specifically on the role of p300/CBP, as a non-histone protein involved in regulating and maintaining epigenetic states in the context of different disease models. They further summarize how small molecule modulators of acetyltransferase as well as other epigenetic enzymes can be exploited for effective therapeutics to treat multiple diseases.

The study by Reddy et al. (Sanjeev Galande and Krishanpal Karmodiya's groups) points out the emergence of a primitive RNA Pol II pausing mechanism for only a subset of genes in the metazoan Hydra before its full manifestation in the bilaterians, such as mouse and humans. Shah et al. (Sanjay Gupta's group) have analysed how histone $\mathrm{H} 2 \mathrm{~A}$ isoforms participate in regulating nucleosome structure and genome plasticity, thereby contributing towards cellular phenotypes and disease.

Gopinath and Kulkarni et al. (Saikrishnan Kayarat's group) have studied the catalytic domain of the S-adenosylL-methionine (AdoMet)-dependent methyltransferase, EcoP15I (a member of the $\beta$ family methyltransferases of the Type III restriction-modification enzyme). The study focusses on the role of an aspartate residue in maintaining the catalytic activity of these diverse yet structurally conserved enzymes. At another scale, Kharerin et al. (Ranjith Padinhateeri's group) propose a physics-based polymer model to investigate the $3 \mathrm{D}$ organization of chromatin responsible for DNA elasticity, the DNA-bending caused by nucleosomes and the linear organization of nucleosomes along DNA. The review by Makowski et al. (Karolin Luger's group) argues for novel models of gene regulation by pioneer transcription factors based on the latest structural and kinetic studies. They advocate for considering the nucleosome as a 'unique structural template'. The review by Kumar et al. (Wendy Bickmore's group) discusses developments in our understanding of how the mammalian genome is organised into compartments, with a focus on the dynamics at the nuclear periphery.

Under physiological conditions cells are exposed to various environmental fluctuations, which alter their energy status. In recent years, several studies have investigated the regulation of transcription driven by key metabolite levels inside the cell, thereby linking the metabolic state of a cell to gene expression. Vadla and Chatterjee et al. (Devyani Haldar's group) report on the dynamics of the histone mark, H3K56Ac upon changes in the metabolic state of mammalian cells (lactate levels) mediated by SIRT6, suggesting the role of environment in regulating histone modifications. Another study, by Rawat and Malhotra et al. (Krishanpal Karmodiya's group), focussing on a Plasmodium falciparum histone acetyltransferase, shows that parasite cellular homeostasis is mediated by nutrient stress conditions. The group goes on to show interaction of the histone acetyltransferase (GCN5) with metabolic enzymes. The review by Lee et al. (Jerry Workman's group) discusses different histone marks which act as 'switches' during the stress of glucose starvation. Maniyadath et al. (Ullas Kolthur's group) review the role of both direct and indirect metabolic inputs towards regulation of gene expression.

Gulati et al. (Vani Brahmachari's group) have identified 38 clusters consisting of arginine methyltransferases, lysine methyltransferases and demethylases in never studied before species of insects. Using Drosophila as a reference genome they look a insects that include pea aphid, triatomid bug, honeybee, silkworm and the red flour beetle. Srinivasan and Mishra (Rakesh Mishra's group) have also analysed the genomes of 10 different species of insects, to find conserved features of PREs (Polycomb Response Elements). Both of these studies contribute towards our understanding of highly conserved epigenetic mechanisms. The perspective from Jongmin Kim and Robert E. Kingston (Robert Kingston's group) on the Polycomb Repressive Complex 1 (PRC1) family discusses recent data suggesting a link between the mechanisms that repress and the mechanisms that generate epigenetic memory. The authors suggest that chromatin compaction and phase separation by PRC1 complexes may allow for such linkage. The perspective from Geeta Narlikar addresses the potential of phase-separation processes to drive and regulate chromatin organization within the nucleus. 


\section{Acknowledgements}

This special issue would not have been possible without the help of many. We would especially like to thank Prof. BJ Rao (Editor-in-Chief, Journal of Biosciences) for promptly agreeing to bring out a special issue on chromatin biology and epigenetics and continued support. We sincerely thank all the reviewers who followed the timelines and provided their critical assessments as well as valuable suggestions towards improving the manuscripts. Dr Ashwin Kelkar and Ankita Sharma from IISER Pune volunteered for coordination with the reviewers and managing resources. Sushila Rajagopal from the Journal of Biosciences editorial office who handled this special issue is thanked for being very prompt and accommodating. A special thanks to Karolin Luger for sharing the beautiful image of the 'Nucleosome' artwork from her private collection.

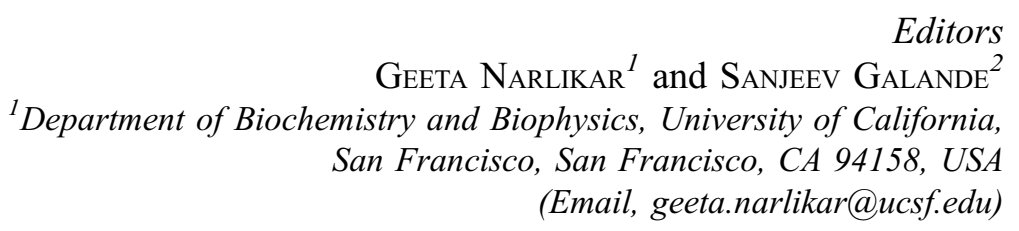

${ }^{2}$ Department of Biology, Centre of Excellence in Epigenetics, Indian Institute of Science Education and Research, Pune 411 008, India

(Email,sanjeev@iiserpune.ac.in) 